\title{
AN ASSESSMENT OF THE METHOD FOR CHANGEPOINT DETECTION APPLIED IN TROPOSPHERIC PARAMETER TIME SERIES GIVEN FROM NUMERICAL WEATHER MODEL
}

\author{
Michal ELIAŠ 1) *, Daniela JARUŠKOVÁ ${ }^{2)}$ and Jan DOUŠA ${ }^{1)}$ \\ 1) Research Institute of Geodesy, Topography and Cartography, Ústecká 98, 25066 Zdiby, Czech Republic \\ 2) Czech Technical University, Faculty of Civil Engineering, Thákurova 7, 16629 Prague, Czech Republic
}

*Corresponding author's e-mail: michal.elias@pecny.cz

\begin{tabular}{l} 
ARTICLE INFO \\
\hline Article history: \\
Received 14 August 2019 \\
Accepted 22 January 2020 \\
Available online 13 February 2020 \\
\hline
\end{tabular}

\section{Keywords:}

Changepoint detection

Time series homogenization

Numerical weather model

\begin{abstract}
We are discussing changepoint detection in tropospheric parameter time series that occurs in a numerical weather reanalysis model. Our approach applies a statistical method that is based on the maximum value of two sample t-statistics. We use critical values calculated by applying an asymptotic distribution. We also apply an asymptotic distribution to finding approximate critical values for the changepoint position. Experiments on "test" and "real" data illustrate the assumed accuracy and efficiency of our method. The method is assessed by its application to our series after adding synthetic shifts. A total of more than 3,000 original profiles are then analysed within the time-span of the years 1990-2015. The analysis shows that at least one changepoint is present in more than $9 \%$ of the studied original time series. The uncertainty of estimated times achieved tens of days for shifts larger than $9 \mathrm{~mm}$, but it was increased up to hundreds of days in the case of smaller synthetic shifts. Discussed statistical method has potential for suspected change point detection in time series with higher time resolution.
\end{abstract}

\section{INTRODUCTION}

Space geodetic techniques exploiting electromagnetic signals suffer from the atmosphere propagation delay. We recognise two major delays; first, the ionospheric delay, which can be eliminated by carrier-phase combination technique and second, the tropospheric delay, usually eliminated by models including physical and meteorological aspects.

The zenith tropospheric delay (ZTD - meaning the signal delay is coming from the zenith direction of the antenna) is usually described as consisting of two contributions: zenith hydrostatic delay (ZHD) that is later defined by the equation (2) and given as a function of the atmospheric pressure, and zenith wet delay (ZWD, see (3)). Although representing only about $10 \%$ of the ZTD, the ZWD plays the most important role in calculating the tropospheric delay for Global Navigation Satellite Systems (GNSS) signals as it cannot be effectively predicted, Bevis et al. (1992). The reason is that the ZWD depends mostly on the distribution of temperature and water vapour in the atmosphere which is a highly spatio-temporally variable. Besides, ZWD is interesting variable from a climatological point of view in the sense that integrated water vapour (IWV) content can be given as a function of ZWD, Bevis et al. (1994).

Some traditional models, like that of Hopfield (1969) or Saastamoinen (1972) were developed for precise ZHD calculation, and even more models were developed for calculating ZWD, as it is a more complex parameter. The most accurate and widelyused was developed by Askne and Nordius (1987). It is an analytical model based on knowledge of the mean temperature, temperature lapse rate, partial water vapour, and its exponential decay rate. Its enhancements through an optimal combination of water vapour and ZWD decay rates has been developed by Dousa and Elias (2014).

The calculation of tropospheric delays depends on input meteorological variables along with the signal trajectory. These are usually not available for real-time GNSS applications and the so-called blind tropospheric models are thus often generated to provide tropospheric corrections, e.g. Lagler et al. (2013). Such models are based on a temporal approximation of selected meteorological parameters, usually given as the result of the time series analysis. Nowadays, Numerical Weather Model (NWM) reanalysis plays an important role as an input source of meteorological data for providing 1) a priori values for ZHD or ZWD parameters, De Haan (2008); 2) coefficients of mapping functions used for converting ZHD and ZWD into slant hydrostatic and wet path delays of actual signal elevations, Boehm et al. (2006); 3) inputs for the conversion of ZWD into IWV content, Nykiel et al. (2018); 4) reference time series in process of changepoint detection in GNSS products, Alshawaf et al. (2017). 
In this context, we would like to emphasise that the long-time series of NWM data used, for the blind model development, for example, can be affected by changepoints, which may degrade the model's accuracy or reliability over time. The changes in the time series may result from satellite data calibrations or from changes in satellite constellations. We thus suggest checking input data of the NWM reanalysis for the occurrence of inhomogeneities, which is the main interest of the paper. For this purpose, we applied a widely used method of mathematical statistics.

Statistical methods of changepoint detection represent a helpful instrument for discovering the discontinuities in the mentioned series. Statistical inference usually consists of two major steps; first, a decision whether the analysed series includes any suspected time of change and second, an estimation of the shifts, Jarušková (1996). In the scope of mathematical statistics, a decision whether the parameter of interest has changed is based on hypothesis testing, see (4). The null hypothesis in this model claims that the process is stationary, while the alternative hypothesis claims that the process is nonstationary. The objective of statistical inference is to decide whether, at some instant, the analysed signal changed its behaviour and started to vary around a different value of mean.

The detection of a change in the series of interest, denoted as $Y_{i}$ where $i \in(1, \ldots, n)$, is made possible by the existence of another series $X_{i}$ for $i \in(1, \ldots, n)$, which can be relied on to be correct and is analogous to the series $Y_{i}$. The series $\left\{Y_{i}\right\}$ is then called a reference series. This approach was recommended by Potter (1981) for detecting shifts in the mean of precipitation series. The same idea of finding inhomogeneities in the series $\left\{Y_{i}\right\}$ using the reference series $\left\{X_{i}\right\}$ was also discussed in the papers Alexandersson and Moberg (1997), Jarušková (1997) or Peterson et al. (1998). A crucial book that covers statistical method used for the purposes pursued in this paper is Csörgő and Horváth (1997).

Homogenization of atmospheric time series are discussed in Vay et al. (2009) where authors studied jumps in the PW (precipitable water) time series. Ning et al. (2016) analysed change point detection method applied on atmospheric water vapor time series that were obtained from the GNSS reprocessed data. The paper authored by Van Malderen et al. (2017) studies some homogenization algorithms efficiency when algorithms were applied on synthetic difference time series between the IGS repro 1 and ERA Interim IWV datasets.

It should be noted that almost all the commonly used methods are suggested for annual and monthly time series. In the case of a time series with a higher time resolution of the studied parameter, some methodologies such as those published in Toreti et al. (2012) or Rienzner and Gandolfi (2013) have been discussed. One of this paper's objectives is to study a possible implementation of (4) to our series $\left\{Z_{i}\right\}$, which is recorded with a six-hour time resolution.

The statistical method introduced in this paper represents a principal concept, which will be further enhanced and applied to two major points of interest in our future work. First, analysing the NWM inputs for blind model development. Second, testing the statistical method for changepoint detection in a time series given from high resolution GNSS tropospheric products. In this study, we applied (4) to the data without any gaps, but in the future, we will also use time series with inhomogeneous time spacing. As mentioned above, a reference series that is stationary exists in a situation optimal for changepoint detection. Unfortunately, in our case such a series is not available and we had to choose a different approach, i.e., to detect a change in the recorded series. However, the potential changepoint may be difficult to detect due to the strong seasonality. Therefore, we removed the seasonality before detecting the changepoint, namely, by computing the median corresponding to a given fixed point in the year and subtracting the median series from our data. The median is chosen because it is not sensitive to outliers Rienzner and Gandolfi (2013). In most cases of changepoint detection, the presented method of time series deseasonalising does not affect the process of detection. However, on some very rare occasions, such deseasonalising may slightly affect the changepoint detection and estimation.

\section{DATASET DESCRIPTION}

Reanalysis is a scientific method for developing a homogeneous record of weather and climate changes in time. Observations and a numerical model are combined to generate a synthesised estimate of the state of the Earth's atmosphere. Such reanalysis typically extends over several decades or longer and covers the entire globe in a grid and vertical levels from the Earth's surface to well above the stratosphere. The ERA-Interim product includes the recent global atmospheric reanalysis produced by the European Centre for Medium-Range Weather Forecasts (ECMWF), which provides temporal resolution of six hours for the surface and upper-air parameters covering the troposphere and the stratosphere with a horizontal resolution of approximately $1.0 \times 1.0$ degree, Dee et al. (2011). It has a total of 60 vertical levels, with the top interface level at $0.1 \mathrm{hPa}$. The ERA-Interim covers the period since 1979 and extended forward in time until August $31^{\text {st }}, 2019$.

Figure 1 shows a segment of all nodes for the analysis. The region covers 3,850 grid nodes within a limited domain by its longitude interval $\lambda \in\left\langle-25^{\circ}, 45^{\circ}\right\rangle$ and latitude interval $\varphi \in\left\langle 10^{\circ}, 65^{\circ}\right\rangle$. 


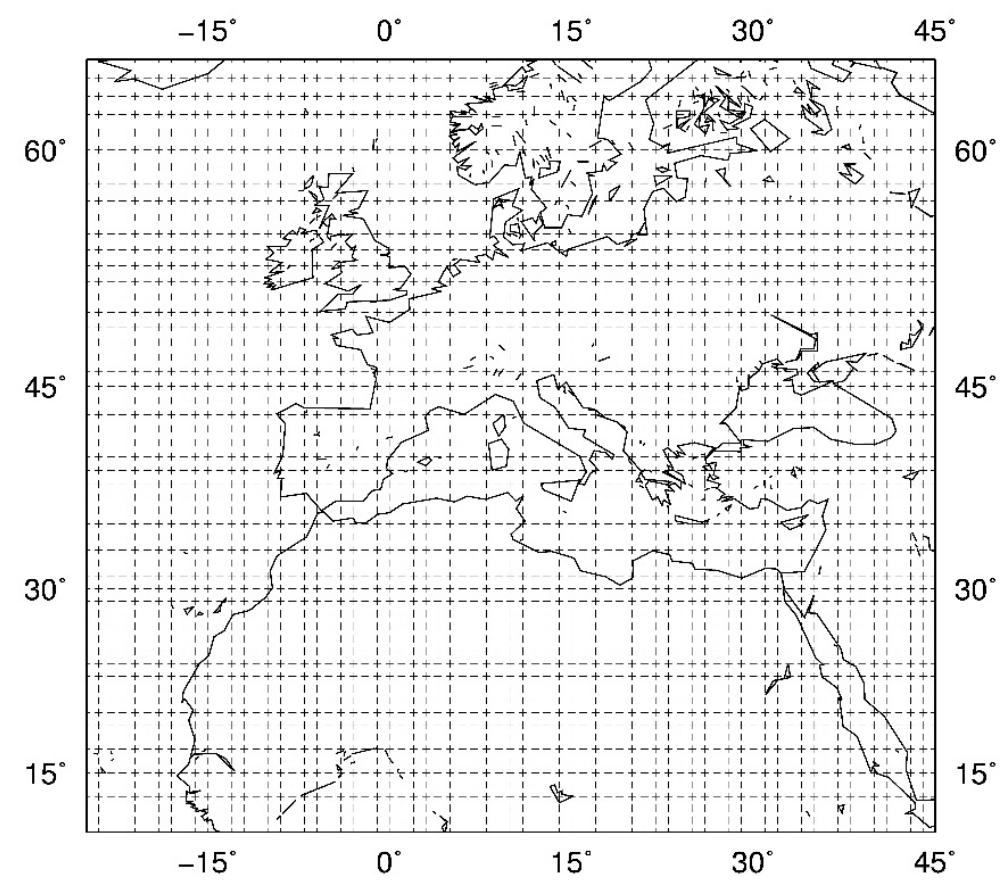

Fig. 1 The locations of analysed grid nodes (cross symbols) within the domain of longitude $\lambda \in\left\langle-25^{\circ}, 45^{\circ}\right\rangle$ and latitude $\varphi \in\left\langle 10^{\circ}, 65^{\circ}\right\rangle$. The region covers 3,850 profiles and data is recorded every six hours within the span of 1990-2015. Each analysed series sample covered more than 36,000 observations.

Even if Figure 1 presents the region covering mostly Europe, our main interest is to cover different climatological and meteorological conditions. The zenith total delay is defined as a sum of the hydrostatic and wet components. Following the paper de Haan (2008), both ZHD and ZWD, which are the parameters of interest in this paper, are formally defined as integrals limited from the surface height to the top of the atmosphere

$Z T D=Z H D+Z W D=10^{-6} \int_{0}^{p_{s}} k_{1} \frac{R_{d}}{g} d p+10^{-6} \int_{0}^{p_{s}} \frac{R_{d}}{\varepsilon g}\left(k_{2}-\varepsilon k_{1}+\frac{k_{3}}{T}\right) q d p$

where $\varepsilon$ is the ratio of gas constants for dry $R_{d}$ and wet $R_{w}$ air; $q$ is the specific humidity; $g$ is the gravity acceleration; $T$ is the temperature; $p_{s}$ is the pressure at the surface height; and finally, the constants $k_{1}, k_{2}$, and $k_{\varepsilon}$ are the so-called refractivity constants. The ZTD can be directly estimated from observations with the aid of various space geodetic techniques, such as GNSS and VLBI (Very Long Baseline Interferometry) and the ZHD can be precisely derived from the atmospheric pressure measurement. The ZWD can then be derived by subtracting the ZHD from the ZTD. If we are using the NWM model as input, it is more convenient to calculate ZTD, ZHD or ZWD by numerical integration of the (1) over the data provided in the model's vertical levels. Using the numerical integration of equation (1), both ZHD and ZWD can be approximated by

$$
\begin{aligned}
& Z H D \approx 10^{-6} k_{1} \frac{R_{d}}{g_{s}}\left(p_{s}-p_{j}\right)+10^{-6} \sum_{i=1}^{N} k_{1} \frac{R_{d}}{g_{i}} \Delta p_{i} \\
& Z W D \approx 10^{-6} \frac{R_{d}}{\varepsilon g_{s}}\left(k_{2}-\varepsilon k_{1}+\frac{k_{3}}{T_{s}}\right) q_{s}\left(p_{s}-p_{j}\right)+10^{-6} \sum_{i=j}^{N} \frac{R_{d}}{\varepsilon g_{i}}\left(k_{2}-\varepsilon k_{1}+\frac{k_{3}}{T_{i}}\right) q_{i} \Delta p_{i}
\end{aligned}
$$

where subscripts $s$ and $j$ denote the values referring to the surface height and the model level, respectively, and $\Delta p_{i}$ is the thickness of the model level $i$, which is defined by $p_{i+1}^{h}-p_{i}^{h}$, see de Haan (2008). 


\section{METHODOLOGY}

\subsection{SEASONALITY PROBLEM AND MEDIAN SERIES DEVELOPMENT}

According to the paper by Moberg and Alexandersson (1997), the series of interest should be compared with a reference series formed of highquality data obtained from several stations where the variations of climate resemble the behaviour of the series at the candidate site, Alexanderson (1986). In our case, such a series is not available. Nevertheless, our series are highly seasonal, and we thus have to remove the seasonal effect. For any fixed time, we take the median of all values over $T$ years that were available at the same time of the year. In this way, a series of $365 \times 4$ elements is created as the NWM model provides four values per day. We call this series the median year series. Finally, we subtract the median year series from our series of interest. A similar approach was applied by Rienzner and Gandolfi (2013), where the authors used highly correlated neighbouring series. In our work, the median series does not cover any observations of the time series from the neighbouring grid nodes. The reason is that the spatial resolution of inputs in the longitude/latitude direction is 1.0 degree, which is approximately equal to $100 \mathrm{~km}$ on the Earth's surface. This fact may cause that the median year series, as suggested by Rienzner and Gandolfi (2013), might be mixed by the values with different climatological or meteorological conditions, and contaminated by errors due to additional spatial interpolations.

\subsection{CONCEPT OF CHANGEPOINT DETECTION METHOD}

In mathematical statistics, a decision whether the series is stationary is based on hypothesis testing, Antoch et al (2002). Our desire is to detect a change in the location parameter of a deseasonalised $\operatorname{series}\left\{Z_{i}\right\}$.

Supposing that the potential changepoint $k$ is unknown, we can define the null hypothesis $H_{0}$ and the alternative $A$ in the following way:

$$
\begin{aligned}
& H_{0}: Z_{i}=\mu+e_{i} \text { for } i=1, \ldots, n \\
& A: \exists k \in\{1, \ldots, n-1\} \text { such that } \\
& Z_{i}=\mu+e_{i} \text { for } i=1, \ldots, \mathrm{k} \\
& Z_{i}=\mu+\delta+e_{i} \text { for } i=k+1, \ldots, n .
\end{aligned}
$$

where $\left\{e_{i}\right\}$ are the zero mean random errors distributed according to the same distribution, $\mu \in \mathbb{R}^{1}$ is the mean value before the changepoint and $\delta \neq 0$ is a shift in the mean value after the changepoint. In such a way, the null hypothesis $H_{0}$ claims that the sequence $\left\{Z_{i}\right\}$ is stationary while the alternative hypothesis $A$ claims that the shift in the mean occurs at a certain unknown time $k$. In the case when $\left\{e_{i}\right\}$ are independent and identically distributed variables distributed according to a normal distribution with variance $\sigma^{2}>0$, we may use the statistic

$T(n)=\max _{k=1, \ldots, n-1}\left|T_{k}\right|$,

where the statistics $\left\{T_{k}\right\}$ are two-sample t-statistics. The statistic $T_{k}$ may be expressed as follows:

$T_{k}=\sqrt{\frac{(n-k) k}{n}}\left(\bar{Z}_{k}-\bar{Z}_{k}^{*}\right) \frac{1}{s_{k}}$

with $S_{k}=\sum_{i=1}^{n} Z_{i}, \quad S_{k}^{*}=\sum_{i=k+1}^{n} Z_{i}, \quad \bar{Z}_{k}=k^{-1} S_{k}$, $\bar{Z}_{k}^{*}=(n-k)^{-1} \bar{S}_{k}^{*}$ and finally

$$
s_{k}^{2}=(n-2)^{-1}\left[\sum_{i=1}^{k}\left(Z_{i}-\bar{Z}_{k}\right)^{2}+\sum_{i=k+1}^{n}\left(Z_{i}-\bar{Z}_{k}^{*}\right)^{2}\right] .
$$

In order to decide whether the null hypothesis should be rejected or not,

$T(n)=\max _{k=1, \ldots, n-1}\left|T_{k}\right|>z_{\alpha}$,

where $z_{\alpha} i$ s an $\alpha \cdot 100 \%$ critical value, we need to know critical values of $T(n)$. For their exact determination, it is necessary to know the distribution of the variables $\left\{Z_{i}\right\}$, Csörgő and Horváth (1997). However, in most cases, even if the distribution of $\left\{Z_{i}\right\}$ is known, the distribution of $T(n)$ is so complex that its critical values can only be calculated for a small number of observations. On the other hand, the real critical values can be approximated by one of the following appropriate methods:

- By applying the Bonferroni inequality, Worsley (1982).

- $\quad$ By simulations, Ning et al. (2016).

- By applying the asymptotic distribution (for large n), Yao and Davis (1986).

In our study, supposing that the number of variables is large, we used approximate critical values that can be calculated from Yao and Davis (1986)

$\left(\max _{1 \leq k \leq n-1}\left|T_{k}\right|>\frac{x+b_{n}}{a_{n}}\right) \approx 1-\exp \left\{-2 e^{-x}\right\}$,

where

$a_{n}=\sqrt{2 \log \log n}$,

$b_{n}=2 \log \log n+0.5 \log \log \log n-0.5 \log \pi$.

We suppose that the null hypothesis was rejected using the hypothesis testing (4), (6). We therefore conclude that a change in the mean occurred. Suppose that the true value of that change is $m$. Denoting $\widehat{m}$, the estimate of the changepoint can be given as

$\widehat{m}=\underset{k=1, \ldots, n-1}{\operatorname{argmax}}\left|T_{k}\right|$.

The $\hat{\mu}$ and $\hat{\delta}$ are then the least squares estimates of $\mu$ and $\delta$ supposing that the change occurred at the time $\hat{m}$. In other words, $\widehat{m}, \hat{\mu}$ and $\hat{\sigma}$ are the values which minimise. 


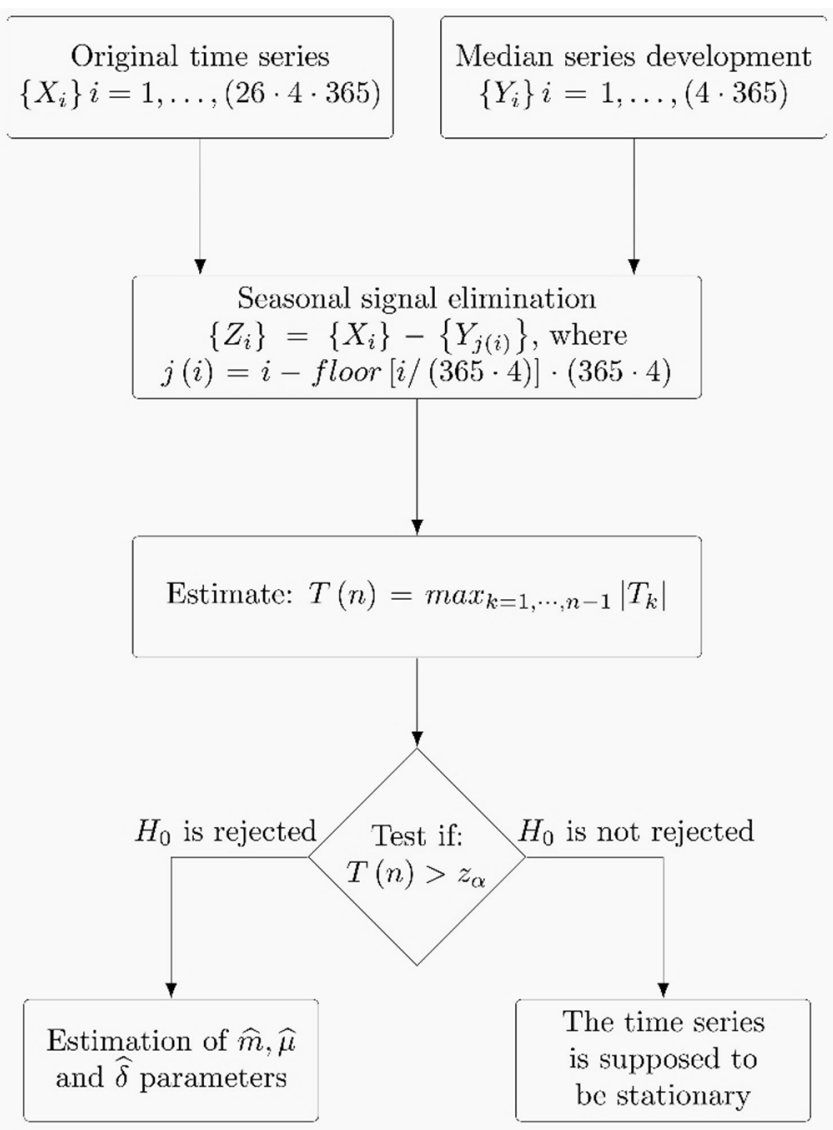

Fig. 2 The scheme of the changepoint detection in studied ZHD and ZWD time series based on the maximum value of two sample t-statistics.

$\sum_{i=1}^{k}\left(Z_{i}-\mu\right)^{2}+\sum_{i=k+1}^{n}\left(Z_{i}-\mu-\delta\right)^{2}$,

and let $\hat{\sigma}^{2}$ be an estimate of $\sigma^{2}$

$\hat{\sigma}^{2}=s_{\widehat{m}}^{2}=\frac{1}{n-2}\left\{\sum_{i=1}^{\widehat{m}}\left(Z_{i}-\bar{Z}_{\widehat{m}}\right)^{2}+\sum_{i=\widehat{m}+1}^{n}\left(Z_{i}-\bar{Z}_{\widehat{m}}^{*}\right)^{2}\right\}$.

\subsection{SCHEME OF METHOD APPLICATION}

To produce the extended median series $\left\{Y_{j(i)}\right\}$, it is necessary to add an appropriate median value $\left\{Y_{j}\right\}$ to time of the original time series $\left\{X_{i}\right\}$, which is characterized by index $i$. The whole procedure of changepoint detection is shown in Figure 2.

A visual presentation for one of the selected node profiles, together with the median year series, is presented in Figure 3. The upper panel of Figure 4 displays the deseasonalised time series $\left\{Z_{i}\right\}$, and the bottom one presents the statistics $\left\{\left|T_{k}\right|\right\}$, together with its critical value at the 0.05 significance level. This pair of Figures shows the time series with a synthetic shift of $15 \mathrm{~mm}$ introduced at "2004-09- 30 18:00:00" added for testing the accuracy of the method and its practical presentation. The node position is: $\varphi=50^{\circ}$ and $\lambda=-15^{\circ}$, see Table 1 . The time series consists of more than 36,000 records within the time-span of "1990-01-01 00:00:00"- "2015-12-31 18:00:00".

The maximum of the statistics $\left\{\left|T_{k}\right|\right\}$ was identified at "2004-11-18 00:00:00" and the value was equal to 27.47. Comparing this value with the $5 \%$ critical value 10.51 , the null hypothesis defined in (4) is rejected at the 0.05 significance level. Detecting the changepoint in the presented series, the question of the presence of another changepoint may arise. However, this question is not the primary goal of this paper and it will be studied in the future. A possible way would be to split the time series into two parts and study them separately, i.e., to consider independently the parts before and after the changepoint.

\section{EXPERIMENTAL RESULTS}

We applied the presented statistical method to the changepoint detection procedure in the time series of ZHD and ZWD derived from the NWM model. In this section, we present the results of that practical experiment. 


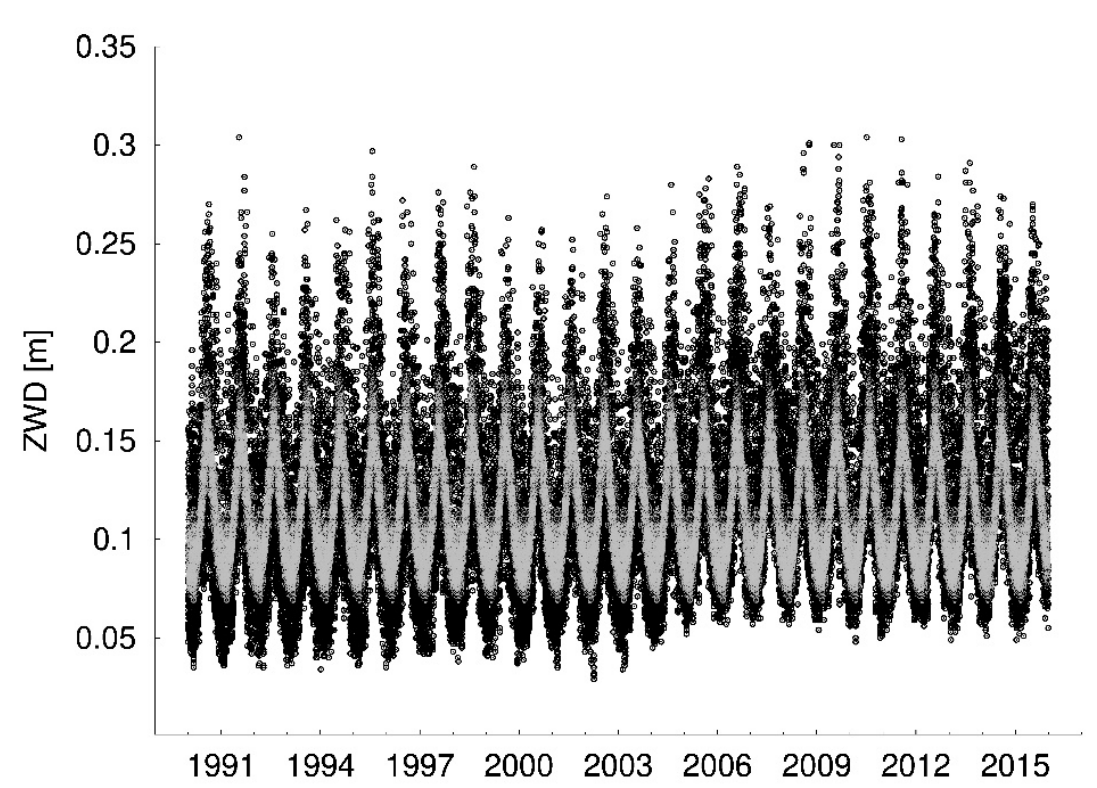

Fig. 3 Example of the ZWD time series of interest (black) and the median year series (silver) that is developed from the candidate time series. Synthetic shift of $15 \mathrm{~mm}$ was introduced at "2004-09-30 18:00:00".
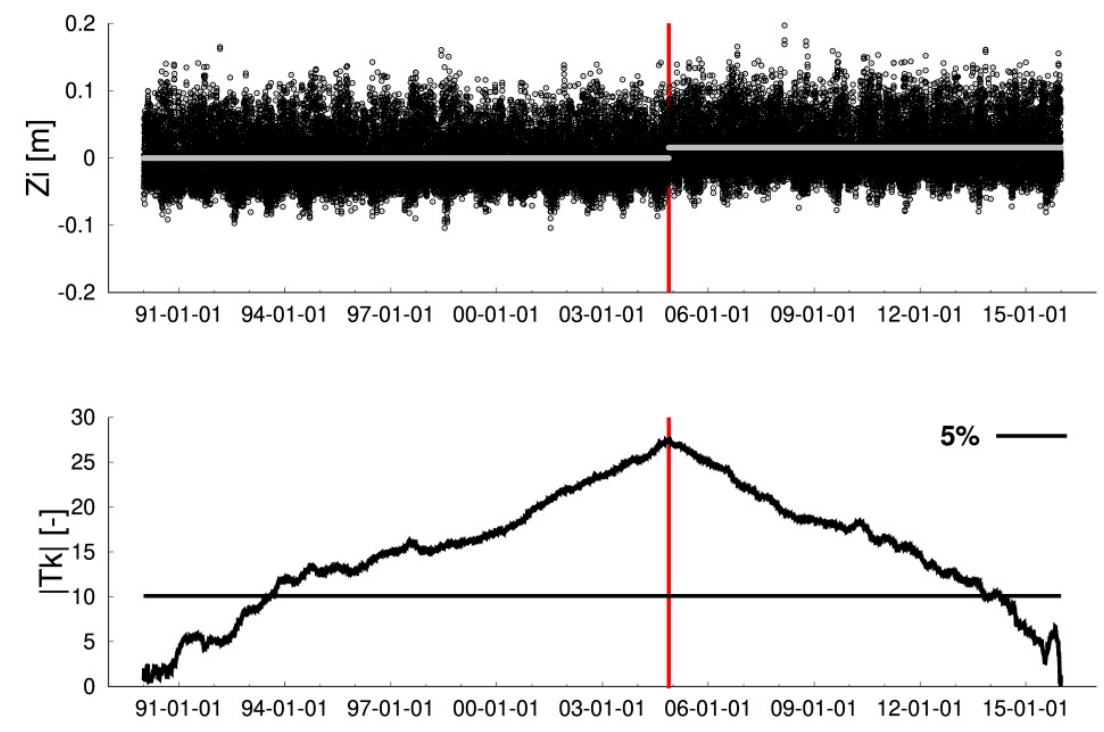

Fig. 4 Example of changepoint detection based on applying the $\left|T_{k}\right|$ statistic. Upper panel of the presented figure shows deseasonalised time series as well as the adjusted means and the bottom panel shows the progress of the $\left|\mathrm{T}_{\mathrm{k}}\right|$ statistics. The vertical red line refers to the changepoint location.

The whole experiment is divided into two scenarios using the testing (Test) and real (Real) data as defined in Table 1.

First, we evaluated the method suitability through the analysis of semi-synthetic data (Test blocks) based on the original time series from the ERA-Interim and modified by introducing shifts according to Table 1 . The Test blocks were defined as squares consisting of $11 \times 11$ nodes in the regions where no changepoints were initially detected. All Test nodes were modified in the same time of 2004-
09-30 18:00:00 (MJD 53278.75). The main objective of this experiment is to statistically assess the detected times and values of changepoint and compare the results with the known ones.

Second, we statistically assessed the changepoint detection in the original time series when searching for real (unknown) changepoints. For this purpose, we defined rectangular blocks denoted as Real in Table 1, and we assessed consistencies and uncertainties of times and values of individually detected changepoints within each block. 
Table 1 List of all tested (Test and Real) blocks. The Test blocks were defined as squares consisting of 121 nodes in the regions where no changepoints were initially detected. The last column then includes information of the added synthetic shift in the blocks at the instant of 2004-09-30 18:00:00 (Modified Julian Date - MJD is 53278.75).

\begin{tabular}{lccrrr}
\hline Case & \multicolumn{2}{c}{ Latitude $\left[^{\circ}\right]$} & \multicolumn{2}{c}{ Longitude $\left.{ }^{\circ}\right]$} & Shift \\
\cline { 2 - 5 } Study & $\varphi_{\min }$ & $\varphi_{\max }$ & $\lambda_{\min }$ & $\lambda_{\max }$ & {$[\mathbf{m m}]$} \\
\cline { 2 - 5 } Test 1 & 45 & 55 & -20 & -10 & 15 \\
Test 2 & 45 & 55 & -8 & -2 & 12 \\
Test 3 & 45 & 55 & 4 & 14 & 9 \\
Test 4 & 45 & 55 & 16 & 26 & 6 \\
Test 5 & 45 & 55 & 28 & 38 & 3 \\
Real 1 & 57 & 62 & 9 & 19 & - \\
Real 2 & 34 & 42 & 13 & 23 & - \\
Real 3 & 33 & 37 & 27 & 34 & - \\
Real 4 & 26 & 30 & 20 & 33 & - \\
Real 5 & 10 & 23 & 5 & 40 & - \\
Real 5a & 14 & 22 & 11 & 17 & - \\
Real 5b & 10 & 14 & 8 & 21 & - \\
Real 5c & 13 & 18 & 22 & 30 & - \\
Real 5d & 14 & 19 & 34 & 36 & - \\
\hline
\end{tabular}

\subsection{CASE STUDY: CHANGEPOINT DETECTION IN "TEST" BLOCKS}

Figure 5 shows the results of detected changepoints when displaying their times given as Modified Julian Days. Table 2 then contains summary statistics including the total number of grid points with identified changepoints. The common expressions of BIAS and RMSE are

$B I A S=\frac{1}{N} \sum_{i=1}^{N} T_{i}-T^{0}$,

$R M S E=\sqrt{\frac{1}{N} \sum_{i=1}^{N}\left(T_{i}-T^{0}\right)^{2}}$,

where $\left\{T_{i}\right\}$ are estimated changepoints or the shifts in our study, and $T^{0}$ is the reference value. Figure 6 shows the distribution of the estimated shifts in the time series, and the summary statistics are then given in Table 3. We can observe a good agreement between the estimated and true times of the synthetically introduced shifts, in particular for those larger than $6 \mathrm{~mm}$. The RMSE indicates uncertainties of 81 and 89 days for a shift of $15 \mathrm{~mm}$ and $12 \mathrm{~mm}$, respectively. The uncertainty of these estimates increases with the decrease of the shift magnitude, indicating a quarter of year or more for shifts below $10 \mathrm{~mm}$.

In the paper Antoch et al. (1997) the behaviour of the selected statistical methods which are based on the partial sum were discussed. In addition to others, they showed some of them are appropriate for the change detection at the beginning or at the end of the series, while others are more powerful when the suspected time of the change is in the middle of the series. We dealt with this issue as well. Table 4 summarises times of adding synthetic shifts into the original time series. These times were distributed over the whole time-span of the candidate time series. The principle of the testing was the same as the one presented in the previous example. For each synthetic changepoint time, five tested blocks were defined following the scheme shown in Figures 5, 6 and Table 1.

Table 2 Statistics of changepoints detected in semisynthetic time series. The RMSE indicates uncertainties of 81 and 89 days for a synthetic shift of $15 \mathrm{~mm}$ and $12 \mathrm{~mm}$, respectively.

\begin{tabular}{cccccc}
\hline Block & No. & $\begin{array}{c}\text { Mean } \\
\text { [MJD] }\end{array}$ & $\begin{array}{c}\text { BIAS } \\
\text { [day] }\end{array}$ & $\begin{array}{c}\text { SDEV } \\
\text { [day] }\end{array}$ & $\begin{array}{c}\text { RMSE } \\
\text { [day] }\end{array}$ \\
\hline Test 1 & 121 & 53338.26 & 59.51 & 54.84 & 80.62 \\
Test 2 & 121 & 53269.25 & -9.50 & 89.66 & 89.42 \\
Test 3 & 121 & 53382.49 & 103.74 & 188.51 & 213.80 \\
Test 4 & 121 & 53685.77 & 407.02 & 276.75 & 490.91 \\
Test 5 & 55 & 53506.00 & 227.25 & 367.09 & 426.03 \\
\hline
\end{tabular}

Table 3 Statistics of shifts estimated in Test blocks. We can observe relatively good agreement between the estimated and synthetically introduced shifts.

\begin{tabular}{cccccc}
\hline Block & No. & $\begin{array}{c}\text { Mean } \\
{[\mathbf{m m}]}\end{array}$ & $\begin{array}{c}\text { BIAS } \\
{[\mathbf{m m}]}\end{array}$ & $\begin{array}{c}\text { SDEV } \\
{[\mathbf{m m}]}\end{array}$ & $\begin{array}{c}\text { RMSE } \\
{[\mathbf{m m}]}\end{array}$ \\
\hline Test 1 & 121 & 15.68 & 0.68 & 0.53 & 0.86 \\
Test 2 & 121 & 13.00 & 1.00 & 0.63 & 1.17 \\
Test 3 & 121 & 11.51 & 2.51 & 0.57 & 2.57 \\
Test 4 & 121 & 9.84 & 3.84 & 0.63 & 3.89 \\
Test 5 & 55 & 8.0 & 5.03 & 0.77 & 5.09 \\
\hline
\end{tabular}

Figure 7 presents percentages of successfully detected changes in each of the tested blocks as depending on the instants of the shifts within the data span (x-axis), see Table 4. The black column in the Figure represents the number of detected changepoints in the time series, where a synthetic shift of $15 \mathrm{~mm}$ was added. The legend further distinguishes other case studies, i.e., for shifts $12,9,6$ and $3 \mathrm{~mm}$. We clearly see a higher success rate for detecting changepoints located in the middle of the series compared to the interval borders. For example, the method's efficiency of $3 \mathrm{~mm}$ shift detection reaches about $50 \%$. The success of $100 \%$ is observed for $6 \mathrm{~mm}$ shifts and larger, if the time of the change occurred 3-4 years

Table 4 Times of synthetic shifts introduced in time series. Introduced synthetic changepoints are distributed over the whole time-span of analysed time-series.

\begin{tabular}{ccc}
\hline ID. & Synthetic changepoint & MJD \\
\hline $\mathbf{1 9 9 1}$ & $1991-07-06$ 18:00:00 & 48443.75 \\
$\mathbf{1 9 9 8}$ & $1998-07-06$ 18:00:00 & 51000.75 \\
$\mathbf{2 0 0 4}$ & $2004-09-3018: 00: 00$ & 53278.75 \\
$\mathbf{2 0 1 0}$ & $2010-06-1818: 00: 00$ & 55365.75 \\
$\mathbf{2 0 1 5}$ & $2015-01-0100: 00: 00$ & 57023.00 \\
\hline
\end{tabular}




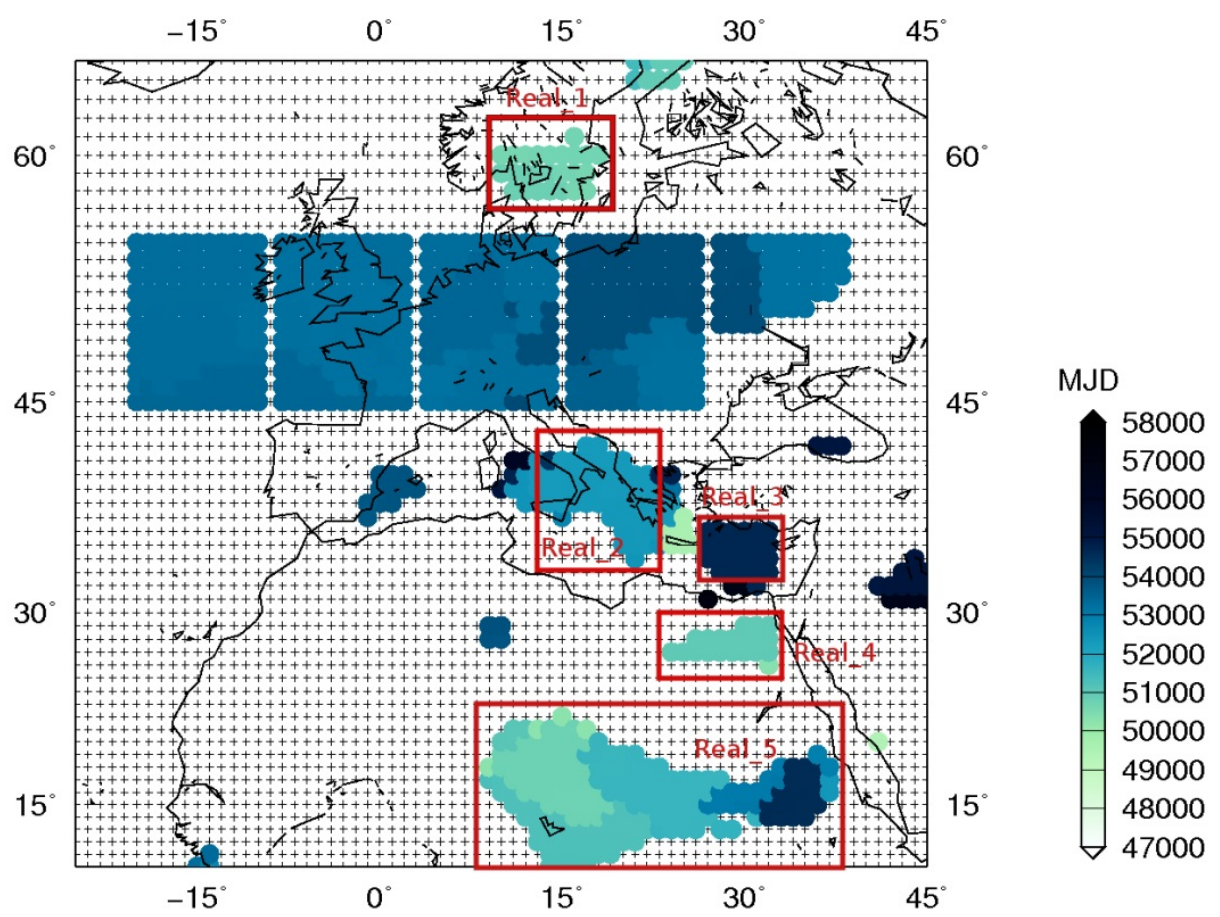

Fig. 5 Spatial distribution of detected changepoints. Red boxes show Real blocks defined for the discussed method assessment.

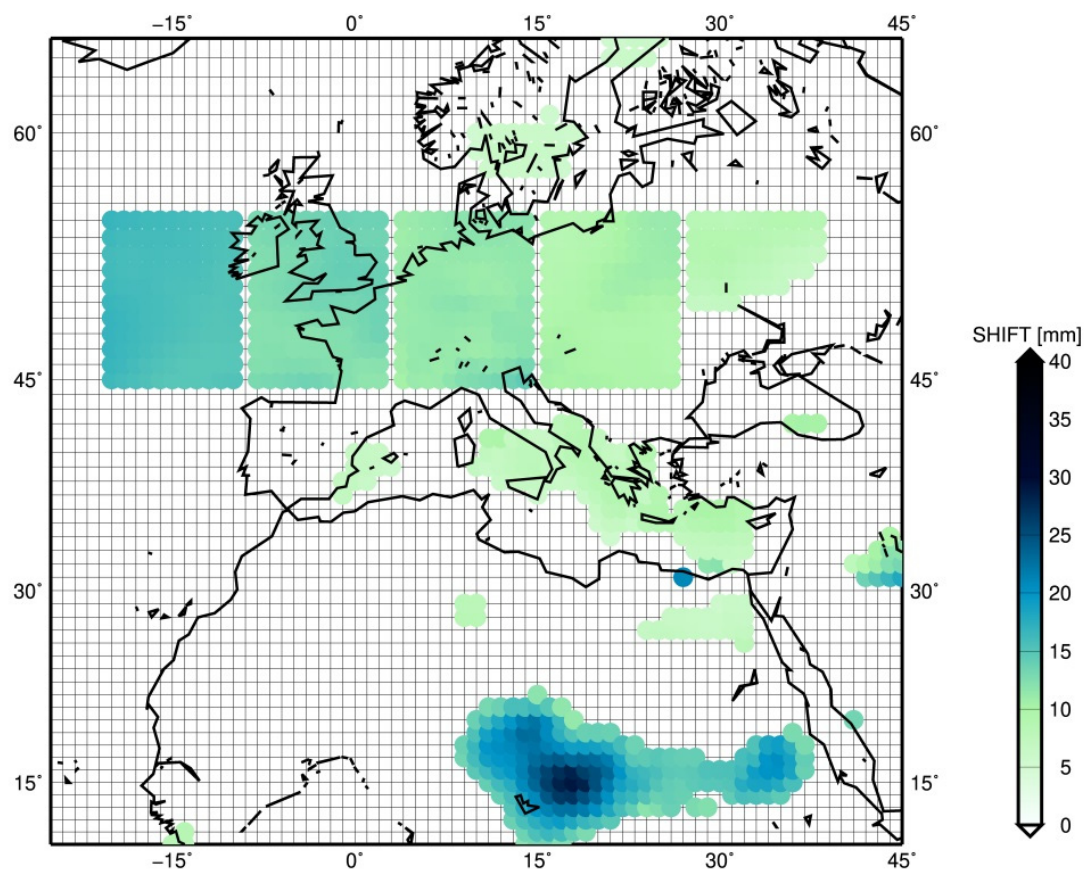

Fig. 6 Spatial distribution of estimated shifts. The most significant shifts were mostly estimated in North Africa and Mediterranean Sea. Several blocks were also detected in southern Sweden. 


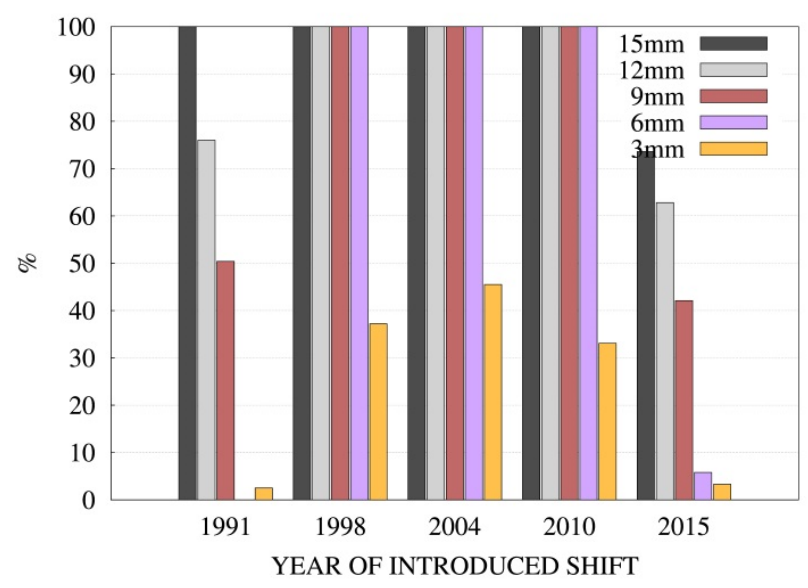

Fig. 7 Statistics of successfully detected synthetic shifts in the time series

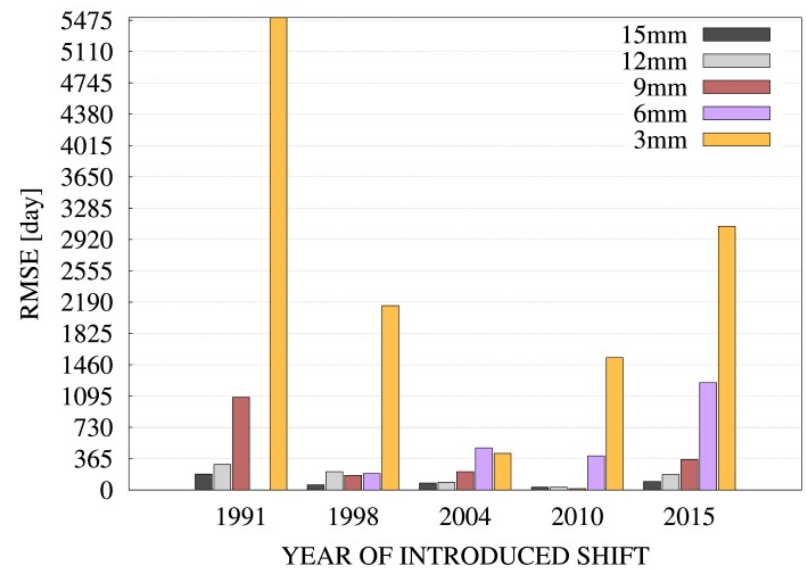

Fig. 8 Statistics of changepoint detection uncertainty.

away from the interval borders, i.e., roughly about $1 / 5$ of the entire interval. The efficiency of detecting relatively small shifts, however, rapidly decreases when the suspected time occurs near the interval borders. For synthetic shifts of $9 \mathrm{~mm}$, the efficiency drops below $50 \%$.

Figure 8 shows RMSE characterising the method's accuracy for each of the analysed case studies. We can see relatively small errors when the suspected time of change is in the middle of the series. Figure 8 also demonstrates that the degree of the statistical uncertainties is significantly smaller for larger shifts.

Finally, Figure 9 shows estimated values of the BIAS, standard deviation and RMS error. The results correspond to the case study while adding the synthetic shift of $9 \mathrm{~mm}$. The best results are clearly observed when the change was synthetically added in the middle of the series. We can notice higher error rates at the beginning of the series compared to the end of the same sample.

Based on the results of semi-synthetic time series analysis, we can conclude that the homogeneous time series, defined as the median year series in combination with the applied statistical method of the changepoint detection, are reliable for detecting at least one changepoint in the candidate time series. The applicability of the reference series, provided as the median year series, will be further studied in the following work, in particular when considering the use for non-equidistant time series, such as given by GNSS ZTD products.

\subsection{CASE STUDY: CHANGEPOINT DETECTION IN "REAL" BLOCKS}

Figure 5 provided a view on changepoints detected in the original time series grouped in REAL blocks. As we know nothing about potential changes, we can only study a consistency of detected changepoint times within the defined blocks provided that changepoints in the original NWM time series should be similar in specific domains. Indeed, the grid nodes with statistically proven changes are generally distributed in specific areas. We can observe similar characteristics in terms of times and shifts of the detected changepoints. The largest block of changepoints is in the North of Africa. Other interesting regions can be found in the Mediterranean Sea and a small block is also visible in southern Sweden. 


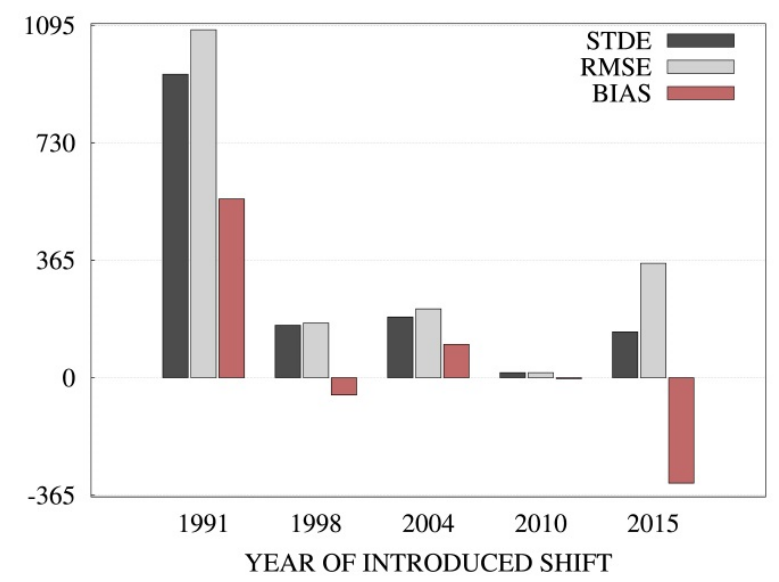

Fig. 9 Statistics of changepoint detection from the analysis of Test 3 block with a $9 \mathrm{~mm}$ synthetic shift introduced to all node time series.

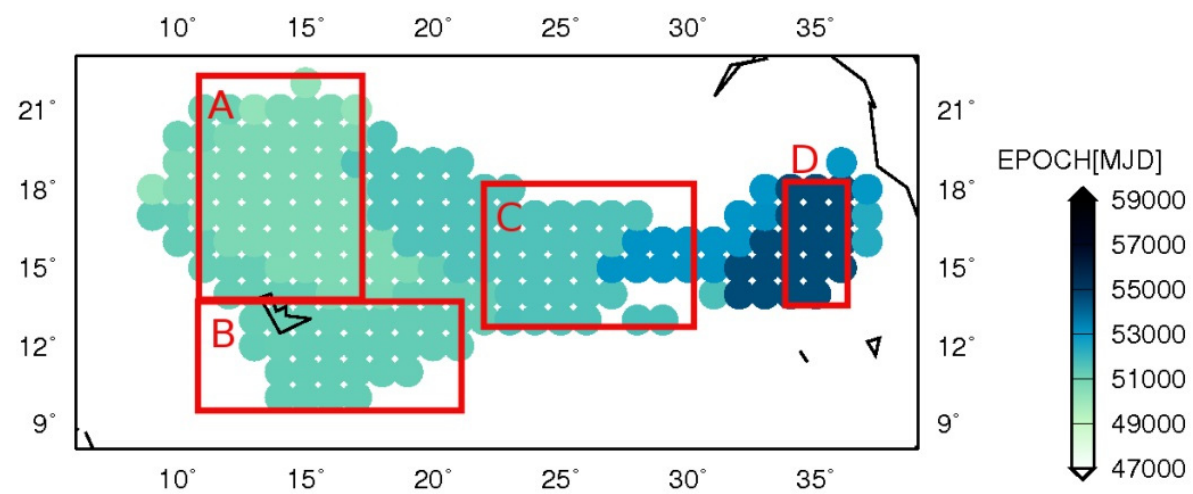

Fig. 10 Distribution of detected changepoint's time in Real 5 block. Sub-regions for specific statistics are displayed in red boxes.

Figure 6 displays mean shifts statistically detected in the original time series and reaching absolute values within a range of approximately 5$20 \mathrm{~mm}$. According to Table 1, the uncertainty of detecting and estimating synthetic shifts of $9 \mathrm{~mm}$ (TEST 3 block). These roughly correspond to the majority of values detected in the original time series, and showed a variation of about 189 days, see Tables 2 and 3. We can assume a similar uncertainty of detecting a common changepoint location within REAL blocks, i.e., without knowing the deviation from the truth.

Table 5 contains statistics of the mean and standard deviation of the detected changepoints times and estimated shifts for each defined block. The number of nodes contributing to the statistics is given in the second column, ranging from 20 to 58. Some blocks show a reliable changepoint detection, which is characterised by a low standard deviation in both estimated quantities, in particular for the shift estimates. However, detecting the shifts reached larger uncertainty in most cases according to the expectation from the analyses of semi-synthetic time series.

Special attention should be paid to the largest block (REAL 5) located in the East Sahara and split into four parts (A-D) due to a clear variability in its estimated changepoint characteristics. Figures 5 and 10 show the detected time changing from east to west, and Figure 6 displays that shifts are particularly increasing towards the two central areas. Statistical assessment is thus difficult for this block, in particular for uncertainties. By splitting the REAL 5 block into four parts, and still using (14)38-56 samples, reduced the standard deviation values of the changepoint times by a factor of 4.7, 8.6, 2.0 and 106.2 for parts A, B, C and $\mathrm{D}$, respectively. Mean shift uncertainties also decreased and, generally, they showed values lower by an order in all blocks indicating the statistical significance of the estimated shifts. According to Table 5, the most significant variability, besides the whole REAL 5 variance, is clearly visible in the case of REAL 5C block.

\section{CONCLUSIONS}

The presented study is focused on two main aspects related to the ZTD time series analysis. First, the applicability of the statistical method for a changepoint detection, which was performed by applying the method on semi-synthetic time series including control change points. Second, we assessed 
Table 5 Statistics of changepoint detected in defined Real blocks. The second column indicated the total number of detected changepoints within the block.

\begin{tabular}{|c|c|c|c|c|c|}
\hline \multirow[b]{2}{*}{$\begin{array}{l}\text { Case } \\
\text { Study }\end{array}$} & \multirow[b]{2}{*}{ No. } & \multicolumn{2}{|c|}{ Changepoint } & \multicolumn{2}{|c|}{ Shift } \\
\hline & & $\begin{array}{l}\text { Mean } \\
\text { [MJD] }\end{array}$ & $\begin{array}{l}\text { Sdev } \\
\text { [day] }\end{array}$ & $\begin{array}{l}\text { Mean } \\
{[\mathrm{mm}]}\end{array}$ & $\begin{array}{l}\text { Sdev } \\
{[\mathrm{mm}]}\end{array}$ \\
\hline Real 1 & 25 & 50698.85 & 78.71 & -5.94 & 0.33 \\
\hline & 8 & 52402 & 460 & -7.40 & 0.85 \\
\hline & & 54690. & & -8.23 & 1.14 \\
\hline & 20 & 50 & 145 & 6.60 & 0.46 \\
\hline & 56 & 50830 & 246.66 & 19.85 & 3.92 \\
\hline & 3 & 5 & 134.82 & 18.04 & 4.13 \\
\hline & 40 & 51837.18 & 573.84 & 15.10 & 1.77 \\
\hline & 14 & 54592.75 & 10.94 & 18.19 & 1.97 \\
\hline Real 5 & 186 & 51690.06 & 1162.05 & 17.90 & 4.01 \\
\hline
\end{tabular}

real time series given from a numerical weather model by applying the same method on searching potential changepoints. The main conclusions of the study can be summarised as follows:

(i) The statistical method for change point detection gives reasonable and applicable results. The strategy assumes removing the seasonality by applying the median year series.

(ii) We analysed 3,850 time series profiles in total and found that the change was suspected to be present in $378(9.8 \%)$ node profiles. Among all detected shifts, $44.4 \%$ and $55.6 \%$ estimated negative and positive values, respectively.

(iii) $\mathrm{We}$ demonstrated a possibility of detecting $100 \%$ control change points of a size larger than $6 \mathrm{~mm}$, and almost $50 \%$ of shifts larger than $3 \mathrm{~mm}$. This is true when change points occur close to the middle of time series in our examples.

(iv) The uncertainty of estimated times achieved tens of days for shifts larger than $9 \mathrm{~mm}$, but it was increased up to hundreds of days in the case of smaller synthetic shifts.

(v) Smaller uncertainties of the change point detection were observed at the end of the series compared to the statistics from change point close to the beginning. The differences are most apparent in instances of smaller shifts. On the other hand, in the case of relatively large shifts, the uncertainties are almost insignificant.

(vi) Figures 5 and 6 show the spatial distributions of the detected changes. Suspected times were mostly detected in North Africa and Mediterranean Sea. Some blocks were also detected in southern Sweden.

(vii) Achieved results are presented for the ZWD time series as there was no change point detected in the case of ZHD's series from the ERA-Interim reanalysis within the study domain. It confirmed our initial expectation since the ZHD is related to the atmospheric pressure, and represents a smoother parameter, easier to be observer and modelled. On the other hand, the ZWD time series strongly depends on satellite observations in areas such as oceans and deserts. These might introduce inhomogeneities due to changes in satellite missions and their calibrations.

The assessment of time series given from the numerical weather model in context of changepoint detection is motivated by their further application. In our next research we will focus on the method's application in the time series given from high resolution GNSS tropospheric records with the presence of inhomogeneous time spacing. We will study an optimal calculation of the reference time series for such a case, including the assessment of the calculation from numerical weather reanalysis. As we plan to use the NWM time series as a reference series it is very important to consider the homogeneity for their further applicability. It is also important to underline that for this study no gaps were involved in the candidate series and each of analysed series sample covered more than 36,000 observations.

\section{ACKNOWLEDGMENTS}

The development of the methodology was supported by the Ministry of Education, Youth and Sports of the Czech Republic (No. LH14089, No. LO1506) and by the European Regional Development Fund No CZ.02.1.01/0.0/0.0/16 019/0000778. The authors kindly acknowledge ECMWF's ERA-Interim reanalysis product and they are also very grateful to the reviewers for their constructive input.

\section{REFERENCES}

Alexandersson, H. and Moberg, A.: 1997, Homogenization of Swedish temperature data. Part I: Homogeneity test for linear trends. Int. J. Climatol., 17, 25-34.

DOI: 10.1002/(SICI)1097-0088(199701)

Alshawaf, F., Balidakis, K., Dick, G., Heise, S. and Wickert, J.: 2017, Estimating trends in atmospheric water vapor and temperature time series over Germany. Atmos. Meas. Tech., 10, 3117-3132.

DOI: 10.5194/amt-10-3117-2017

Antoch, J., Hušková, M. and Prášková, Z.: 1997, Effect of dependence on statistics for determination of change. J. Stat. Plan. Infer., 60, 291-310. DOI: 10.1016/S0378-3758(96)00138-3

Antoch, J., Hušková, M. and Jarušková, D.: 2002, Off-line statistical process control. In: Lauro, C., Antoch, J., Vinzi, V.E. and Saporta, G. (eds): Multivariate total quality control. Contrib. Stat., 1-86. DOI: 10.1007/978-3-642-48710-1_1

Askne, J., Nordius, H.: 1987, Estimation of tropospheric delay for microwaves from surface weather data. Radio Sci., 22, 3, 379-386. DOI: $10.1029 / \mathrm{RS} 022 \mathrm{i003p} 00379$

Bevis, M., Businger, S., Herring, T.A., Rocken, C., Anthes, R.A. and Ware, R.H.: 1992, GPS-Meteorology: 
Remote sensing of atmospheric water vapor using the global positioning system. J. Geophys. Res., 97, 15787-1580. DOI: 10.1029/92JD01517 1

Bevis, M., Businger, S., Chiswell, S., Herring, T.A., Anthes, R.A., Rocken, C. and Ware, R.H.: 1994, GPSMeteorology: Mapping zenith delays onto precipitable water. J. Appl. Meteorol., 33, 379-386.

DOI: $10.1175 / 1520-0450(1994) 033$

Boehm, J., Werl, B. and Schuh, H.: 2006, Troposphere mapping functions for GPS and very long baseline interferometry from European Centre for MediumRange Weather Forecasts operational analysis data. J. Geophys. Res., 111. DOI: 10.1029/2005JB003629

Csörgö, M. and Horváth, L.: 1997, Limit Theorems of Changepoint Analysis, Wiley.

Dee, D. and co-authors: 2011, The ERA-Interim reanalysis: Configuration and performance of the data assimilation system. Q. J. R. Meteorol. Soc., 137, 656, 553-597. DOI: $10.1002 /$ qj. 828

De Haan, S.: 2008, Meteorological applications of a surface network of Global Positioning System receivers. Proefschrift: Wageningen University, Wageningen, $157 \mathrm{pp}$.

Dousa, J. and Elias, M.: 2014, An improved model for calculating tropospheric wet delay. Geophys. Res. Lett., 41, 43894397. DOI: 10.1002/2014GL060271

Hopfield, H.S.: 1969, Two-quartic tropospheric refractivity profile for correcting satellite data. J. Geophys. Res., 74, 4487-4499. DOI: 10.1029/JC074i018p04487

Jarušková, D.: 1996, Changepoint detection in meteorological measurement. Monthly Weather Review, 124, 7, 1535-1543.

DOI: $10.1175 / 1520-0493(1996) 124$

Jarušková, D.: 1997, Some problems with application of changepoint detection methods to environmental data. Environmetrics, 8, 5, 469-483. DOI: 10.1002/(SICI)1099-095X(199709/10)8:5

Lagler K., Schindelegger, M., Boehm, J., Krasna, H. and Nilsson, T.: 2013, GPT2: empirical slant delay model for radio space geodetic techniques. Geophys. Res. Lett., 40, 6, 1069-1073. DOI: 10.1002/grl.50288

Moberg, A. and Alexandersson, H.: 1997, Homogenization of Swedish temperature data. Part II: Homogenized gridded air temperature compared with a subset of global gridded air temperature since 1861. Int. J. Climatol., 17, 35-54. DOI: 10.1002/(SICI)1097-0088(199701)17:1

Ning, T., Wickert, J., Deng, Z., Heise, S., Dick, G., Vey, S., Schöne, T.: 2016, Homogenized time series of the atmospheric water vapor content obtained from the GNSS reprocessed data. J. Climate, 29, 7, 2443-2456. DOI: 10.1175/JCLI-D-15-0213.1

Nykiel, G., Wolak, P. and Figurski, M.: 2018, Atmospheric opacity estimation based on IWV derived from GNSS observations for VLBI applications. GPS Solut., 22, 9. DOI: $10.1007 / \mathrm{s} 10291-017-0675-9$
Peterson, T.C., Easterling, D.R., Karl, T.R., Groisman, P., Nicholls, N., Plummer, N., Torok, S., Auer, I., Boehm, R., Gullett, D., Vincent, L., Heino, R. ,Tuomenvirta, H., Mestre, O., Szentimrey, T., Salinger, J., Førland, E.J., Hanssen - Bauer, I., Alexandersson, H., Jones, P. and Parker, D.: 1998, Homogeneity adjustments of in situ atmospheric climate data: a review. Int. J. Climatol., 18, 1493-1517.

DOI: 10.1002/(SICI)1097-0088(19981115)18:13

Potter, K.W.: 1981, Illustration of a new test for detecting a shift in mean in precipitation series. Mon. Wea. Rev., $109,9,2040-2045$.

DOI: $10.1175 / 1520-0493(1981) 109$

Rienzner, M. and Gandolfi, C.: 2013, A procedure for the detection of undocumented multiple abrupt changes in the mean value of daily temperature time series of a regional network. Int. J. Climatol., 33, 5, 1107-1120. DOI: $10.1002 /$ joc. 3496

Saastamoinen, J.: 1972, Atmospheric correction for troposphere and stratosphere in radio ranging of satellites. The use of artificial satellites for geodesy. Editors: Henriksen, S.W., Mancini, A. and Chovitz, B.H. Washington DC American Geophysical Union Geophysical Monograph Series, 15. DOI: 10.1029/GM015p0247

Toreti, A., Kuglitsch, F.G., Xoplaki, E. and Luterbacher, J.: 2012, A novel approach for the detection of inhomogeneities affecting climate time series. J. Appl. Meteorol. Climatol., 51, 2, 317-326. DOI: 10.1175/JAMC-D-10-05033.1

Van Malderen, R., Pottiaux, E., Klos, A., Bock, O., Bogusz, J., Chimani, B., Elias, M., Gruszczynska, M., Guijarro, J., Zengin Kazanc1, S., and Ning, T.: 2017, Homogenizing GPS integrated water vapour time series: methodology and benchmarking the algorithms on synthetic datasets. Proceedings of the Ninth Seminar for Homogenization and Quality Control in Climatological Databases and Fourth Conference on Spatial Interpolation Techniques in Climatology and Meteorology, Budapest, Hungary, WMO, WCDMPNo. 845, edited by T. Szentimrey, M. Lakatos, L. Hoffmann, 102-114.

Vey, S., Dietrich, R., Fritsche, M., Rülke, A., Steigenberger, P. and Rothacher, M.: 2009, On the homogeneity and interpretation of precipitable water time series derived from global GPS observations. J. Geophys. Res., 114, D10101. DOI: 10.1029/2008JD010415

Worsley, K.J.: 1982, An improved Bonferroni inequality and applications. Biometrika, 69, 2, 297-302. DOI: $10.2307 / 2335402$

Yao, Y.-C. and Davis, R.A.: 1986, The asymptotic behavior of the likelihood ratio statistic for testing a shift in mean in a sequence of independent normal variates. Sankhya, The Indian Journal of Statistics, Series A, 48, 3, 339-353. 